\title{
Cholethorax with biliopleural communication detected on [99mTC]mebrofenin hepatobiliary scintigraphy
}

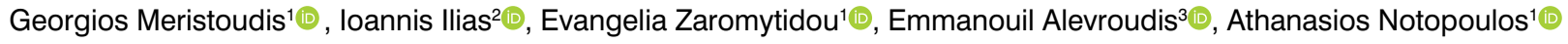
${ }^{1}$ Department of Nuclear Medicine, Hippokration General Hospital, Thessaloniki, Greece 'Elena Venizelou General Hospital, Athens, Greece ${ }^{3}$ Second Department of Radiology, Nuclear Medicine Unit, National and Kapodistrian University of Athens, Attikon University General Hospital, Athens, Greece

[Received 30 III 2021; Accepted 7 V 2021]

\section{Abstract}

Bilious pleural effusion or cholethorax is a rare type of exudative pleural effusion. Here is presented a case of right-sided cholethorax, in which the direct communication between the pleural effusion with the biliary duct was visible only on the posterior images of dynamic hepatobiliary scintigraphy with [99m Tc]mebrofenin.

KEY words: cholethorax; biliopleural communication; hepatobiliary scintigraphy

Nucl Med Rev 2021; 24, 2: 124-125

Cholethorax (ChT, bilious pleural effusion; biliothorax) is very rare. The authors report a case of right-sided ChT in which the continuity of intrathoracic fluid collection with the biliary ductal system was visualized only in posterior images of dynamic hepatobiliary scintigraphy (HBS) with technetium-99m [ $\left.{ }^{99 m} \mathrm{Tc}\right]$ mebrofenin.

A 51-year-old male with a history of decompensated alcoholic cirrhosis underwent orthotopic liver transplantation. Four weeks after the procedure, the patient presented with right upper abdominal and pleuritic chest pain, mild dyspnoea, and fever. Chest X-ray revealed a new right-sided pleural effusion (confirmed by computed tomography; CT, which also showed unspecific intraabdominal fluid collections, Fig. 1). The patient underwent HBS after intravenous administration of $185 \mathrm{MBq}$ of [99mTc]mebrofenin. Synchronous dynamic imaging in anterior and posterior projections was performed with a dual-head $\gamma$-camera

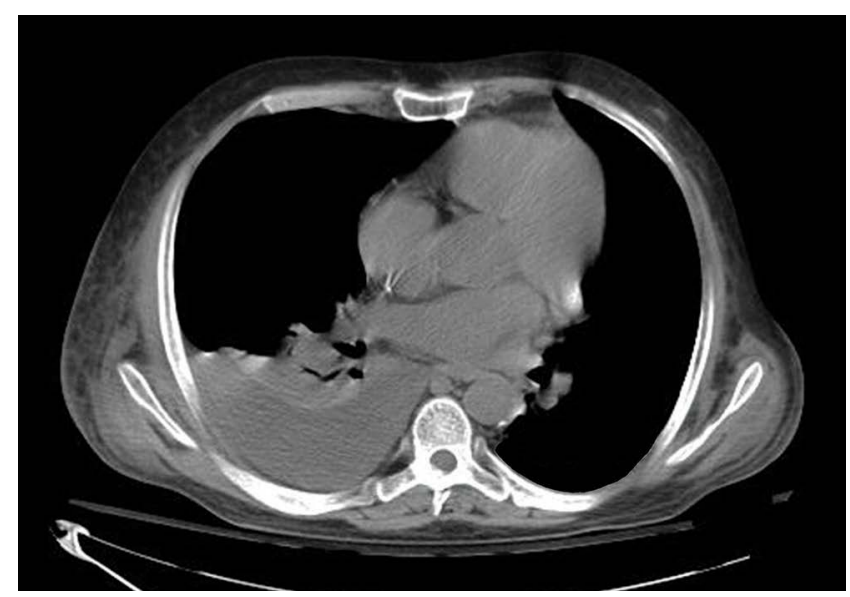

Figure 1. Axial CT image of the lung bases (mediastinal window) shows right pleural fluid collection 

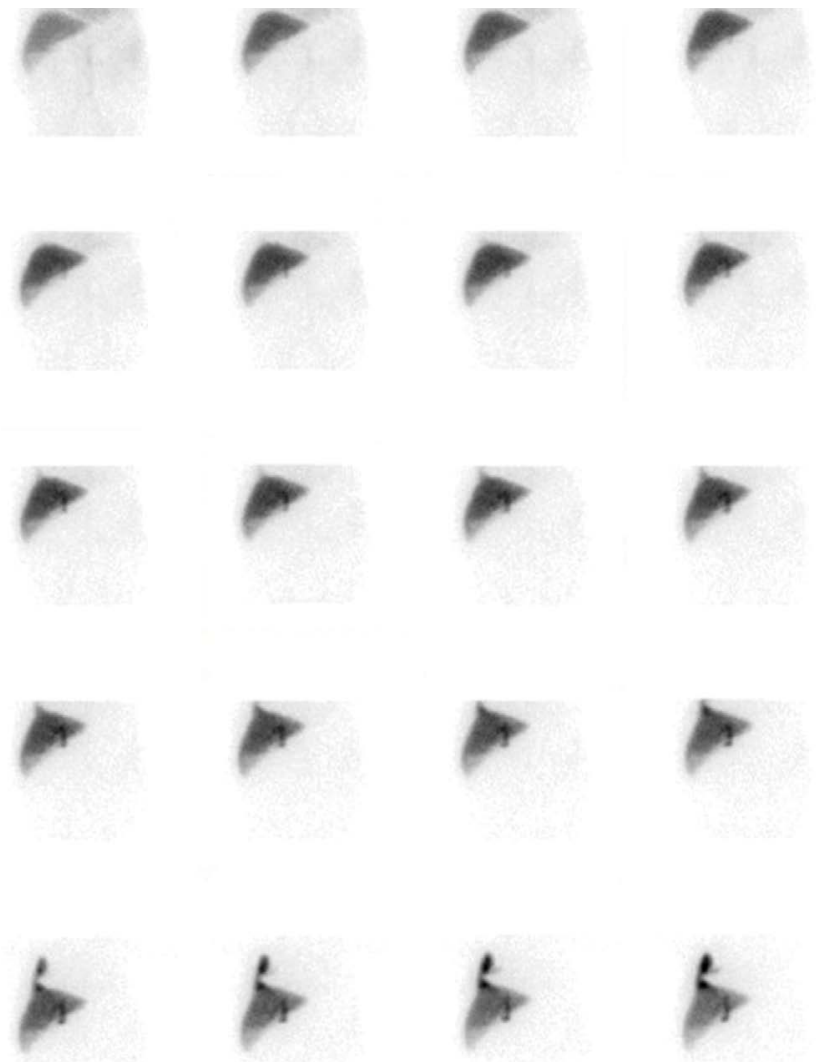

Figure 2. Anterior dynamic images of [ $\left.{ }^{99 \mathrm{mT}} \mathrm{Tc}\right] \mathrm{mebrofenin}$ HBS (reformatted for display at $3 \mathrm{~min} /$ frame) reveal increased tracer activity extending from the superolateral surface of the right hepatic lobe to the right chest. In the last row of images, the findings are much more pronounced in the thorax (which is included in the field of view)

at a rate of $1 \mathrm{~min} /$ frame for $48 \mathrm{~min}$ focused on the abdomen, followed by the second acquisition of dynamic images, to include the entire chest in the field of view, for an additional $12 \mathrm{~min}$. The anterior views revealed a linear area of increased tracer accumulation extending from the superolateral aspect of the right hepatic lobe to the right hemithorax, confirming the diagnosis of ChT. In these views, there was no evidence of a direct connection between the pleural effusion with the biliary tract (Fig. 2). However, the posterior images demonstrated the presence of biliopleural communication, showing radiopharmaceutical emanating from the region of the biliary duct to the superior segments of the right lobe of the liver with subsequent passage into the right chest cavity (Fig. 3). Right-sided thoracentesis was performed; biochemical analysis revealed an exudative pleural effusion with bilirubin at $16.5 \mathrm{mg} / \mathrm{dL}$ (serum $0.5 \mathrm{mg} / \mathrm{dL}$ ),
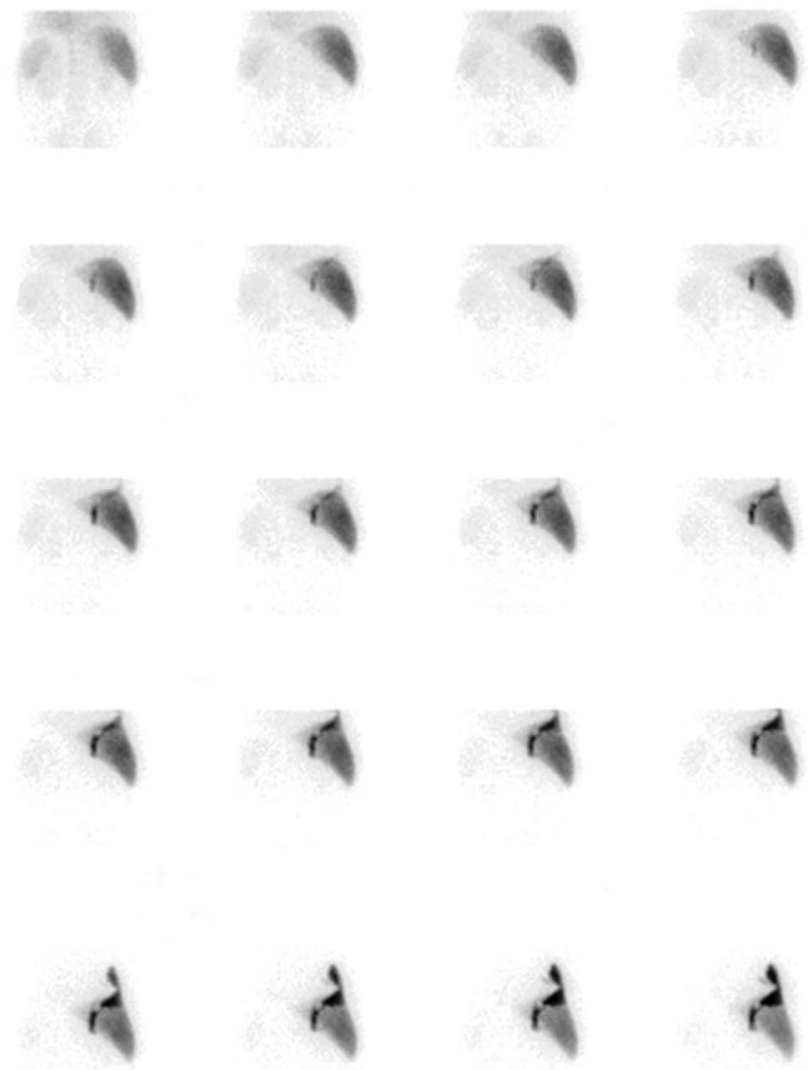

Figure 3. Posterior dynamic images of $\left[{ }^{99 \mathrm{~m} T c] m e b r o f e n i n ~ H B S ~}\right.$ directly delineate the presence of biliopleural communication. Tracer accumulation is clearly visible from the region of the bile duct, ascending to the superior segments of the right hepatic lobe, and extending into the right chest cavity

a finding consistent with ChT. A chest drain was placed, and the patient's symptoms improved.

Despite the rarity of its occurrence, ChT should not be overlooked in the differential diagnosis of patients with pleural effusion, particularly after biliary or hepatic surgery or abdominal trauma. This case illustrates the clinical usefulness of $\left[{ }^{99 \mathrm{~m} T c]}\right.$ mebrofenin HBS for the etiological diagnosis of pleural fluid collection. It also highlights the importance of simultaneous dynamic anterior and posterior imaging, compared with the standard anterior views; in the presented case, the posterior views defined the direct communication between the pleural space with the biliary pathway.

\section{Conflict of interest}

The authors declare no conflicts of interest. 